Z. Klin. Chem. Klin. Biochem.

13. Jg. 1975 , S. $37-40$

\title{
Berechnung der Parameter des Säure-Basen-Haushaltes mit Hilfe eines Kleincomputers*)
}

\author{
Von E. Knoll, H. Wisser und K. Dettmer \\ Abteilung für Klinische Chemie, Robert-Bosch-Krankenhaus, Stuttgart
}

(Eingegangen am 20. März/10. September 1974)

\begin{abstract}
Es wird über die Anwendung und Modifikation eines von Hardt ((1972), Clin. Chem. 18, 658-661) publizierten Programms zur Berechnung des aktuellen $\mathrm{pCO}_{2}$, des Standardbicarbonats und des Basenüberschusses berichtet. Die vergleichende Auswertung von 217 Bestimmungen einer Patientenstichprobe - graphisch mit Hilfe eines Nomogrammes von Siggaard-Andersen und rechnerisch mit Hilfe des Computer-Programms - zeigte eine gute Ubereinstimmung der beiden Auswerteverfahren.
\end{abstract}

\section{Calculation of the parameters of the acid-base balance with the aid of a small computer}

The application and modification of a program published by Hardt ((1972), Clin. Chem. 18, 658-661) for the calculation of the true $\mathrm{pCO}_{2}$, the standard bicarbonate and the base excess are reported. 217 Determinations of a random patient sample were evaluated graphically, with the aid of a nomogram of Siggaard-Andersen, and mathematically with a computer program. There was good agreement between the two evaluation methods.

Die Bestimmung der Parameter des Säure-Basen-Haushaltes ist aus der Notfalldiagnostik nicht mehr wegzudenken. Neben der in jüngster Zeit mehr in den Vordergrund getretenen Direktmessung der entsprechenden Größen, ist die Äquilibriermethode von $A \operatorname{strup}(4,5)$ - Bestimmung mit Hilfe der log $\mathrm{pCO}_{2} / \mathrm{pH}-\mathrm{Beziehung}-$ das z. Zt. noch am häufigsten benützte Verfahren. Die Auswertung erfolgt in der Regel mit Hilfe eines Nomogramms (6). Fallen viele Bestimmungen an, dann ist diese Art der Auswertung relativ zeitaufwendig, und es kommt leicht zu Ablesefehlern. Aus diesem Grunde wurde eine Publikation von Hardt (1) aufgegriffen und ihre Anwendbarkeit in der Routine geprüft. Dem im folgenden beschriebenen Rechenprogramm liegt mit einigen Abänderungen die Arbeit von Hardt (1) zugrunde. Eine Publikation aus jüngster Zeit (7) versucht einen Schritt weiterzugehen und einen Tischcomputer für die Beurteilung der Meßergebnisse heranzuziehen. Die bei Störungen des Säure-Basen-Haushaltes auftretenden Veränderungen des aktuellen $\mathrm{pCO}_{2}$ und Standardbicarbonats werden bei der Erstellung des Diagnoseschlüssels jedoch nicht ausreichend berücksichtigt.

Herleitung der Gleichungen für aktuellen $\mathrm{pCO}_{2}$, Standardbicarbonat und Basenüberschuß

Aus den drei gemessenen pH-Werten $\mathrm{pH}_{\mathrm{a}}$ (aktueller $\mathrm{pH}$ Wert), $\mathrm{pH}_{1}$ (nach Äquilibrierung mit $4 \% \mathrm{CO}_{2}$ ) und $\mathrm{pH}_{2}$

*) Diese Untersuchungen wurden unterstïtzt aus Mitteln der Robert-Bosch-Stiftung, Stuttgart. (nach Äquilibrierung mit $8 \% \mathrm{CO}_{2}$ ) werden folgende Parameter des Säure-Basen-Haushal tes berechnet: Aktueller $\mathrm{pCO}_{2}\left(\mathrm{pCO}_{2}, \mathrm{a}\right)$, Standardbicarbonat und Basenüberschuß. Die zur Erstellung eines Computerprogrammes benötigten Gleichungen für diese drei Parameter wurden in Anlehnung an eine Publikation von Hardt (1) ausgearbeitet und basierten auf dem Säure-Basen-Nomogramm von Siggaard-Andersen. Dabei ist die Gleichung zur Berechnung des Standardbicarbonats eine exakte Wiedergabe des Nomogramms, während die Gleichungen zur Berechnung des $\mathrm{pCO}_{2}$ und des Basenüberschusses Näherungen sind. Zum Äquilibrieren verwenden wir Gasgemische ${ }^{1}$ ) mit $4 \%$ und $8 \%$ Volumenanteil $\mathrm{CO}_{2}$.

Aktueller $\mathrm{pCO}_{2}\left(\mathrm{pCO}_{2}, a\right)$

Der aktuelle $\mathrm{pCO}_{2}$ läßt sich aus folgender Beziehung berechnen (1):

$\frac{\mathrm{pH}_{1}-\mathrm{pH}_{\mathrm{a}}}{\mathrm{pH}_{1}-\mathrm{pH}_{2}}=\frac{\ln \mathrm{pCO}_{2}, 1-\ln \mathrm{pCO}_{2}, \mathrm{a}}{\ln \mathrm{pCO}_{2}, 1-\ln \mathrm{pCO}_{2}, 2}$

Daraus ergibt sich durch Umformen:

$\ln \mathrm{pCO}_{2}, \mathrm{a}=\ln \mathrm{pCO}_{2}, 1+\frac{\mathrm{pH}_{1}-\mathrm{pH}_{\mathrm{a}}}{\mathrm{pH}_{1}-\mathrm{pH}_{2}} \cdot \ln \frac{\mathrm{pCO}_{2}, 2}{\mathrm{pCO}_{2}, 1}$

In dieser Gleichung ist der Ausdruck

$\ln \frac{\mathrm{pCO}_{2}, 2}{\mathrm{pCO}_{2}, 1}=\ln 2=0,693$ konstant, während ln $\mathrm{pCO}_{2}, 1$

1) Firma Hillerkus, Krefeld 
eine Funktion des Barometerdruckes B ist:

$\mathrm{pCO}_{2}=\frac{\left.\mathrm{B}-47^{2}\right)}{100} \cdot \% \mathrm{CO}_{2}$

Trägt man graphisch den $\ln \mathrm{pCO}_{2}, 1$ gegen den Barometerdruck auf, so erhält man zwischen 720 und $800 \mathrm{~mm}$ Hg eine Gerade, die sich durch die Gleichung

$\ln \mathrm{pCO}_{2}, 1=3,35+\frac{0,113}{80}(\mathrm{~B}-760)=\frac{\mathrm{B}+1611,7}{708}$

beschreiben läßt.

Setzt man diese Werte in Gleichung (II) ein, so ergibt sich folgende Beziehung für den aktuellen $\mathrm{pCO}_{2}$ :

$\ln \mathrm{pCO}_{2}, \mathrm{a}=\frac{\mathrm{B}+1611,7}{708}+0,693 \frac{\mathrm{pH}_{1}-\mathrm{pH}_{\mathrm{a}}}{\mathrm{pH}_{1}-\mathrm{pH}_{2}}$

\section{Standardbicarbonat}

Die Berechnung des Standardbicarbonats läßt sich aus der Henderson-Hasselbalch'schen Gleichung ableiten:

$\mathrm{pH}=6,10+\lg \frac{\left[\mathrm{HCO}_{3}^{-}\right]}{40 ; 0,03}$

$\lg \left[\mathrm{HCO}_{3}{ }^{-}\right]=\mathrm{pH}-6,021$

Der pH-Wert in dieser Beziehung ist dabei gegeben durch den Schnittpunkt der $\mathrm{CO}_{2}$-Bindungskurve mit der PlasmaBicarbonat-Skala.

Er läßt sich auf Grund der folgenden Beziehung durch bekannte bzw. gemessene Größen ausdrücken:

$\frac{\mathrm{pH}_{1}-\mathrm{pH}}{\mathrm{pH}_{1}-\mathrm{pH}_{2}}=\frac{\ln 40-\ln \mathrm{pCO}_{2}, 1}{\ln \mathrm{pCO}_{2}, 2-\ln \mathrm{pCO}_{2}, 1}=0,487$
$\mathrm{pH}=-0,487\left(\mathrm{pH}_{1}-\mathrm{pH}_{2}\right)+\mathrm{pH}_{1}$

Setzt man diesen Ausdruck für pH in Gleichung (VII) ein, so erhält man zur Berechnung des Standardbicarbonats folgende Beziehung:

$\lg \left[\mathrm{HCO}_{3}^{-}\right]=-0,487\left(\mathrm{pH}_{1}-\mathrm{pH}_{2}\right)+\mathrm{pH}_{1}-6,021$

\section{Basenüberschuß (BE)}

Bei einem bestimmten $\mathrm{pCO}_{2}, 1(=28,52 \mathrm{~mm} \mathrm{Hg})$ und $\left.\mathrm{pCO}_{2}, 2(=57,04 \mathrm{~mm} \mathrm{Hg})^{3}\right)$ läßt sich die Basenüberschußkurve des Siggaard-Andersen-Nomogramms mit Hilfe einer Taylor-Reihenentwicklung als Funktion der beiden Veränderlichen $\mathrm{pH}_{1}$ und $\mathrm{pH}_{2}$ annähern.

$$
\begin{aligned}
& \mathrm{BE}=\mathrm{F}\left(\mathrm{pH}_{1}, \mathrm{pH}_{2}\right)=\mathrm{F}\left(\mathrm{pH}_{1}{ }^{\prime}, \mathrm{pH}_{2}{ }^{\prime}\right)+\left(\frac{\partial \mathrm{F}}{\partial \mathrm{pH}_{1}}\right)_{\mathrm{pH}_{2}} \\
& \left(\mathrm{pH}_{1}-\mathrm{pH}_{1}{ }^{\prime}\right)+\left(\frac{\partial \mathrm{F}}{\partial \mathrm{pH}_{2}}\right)_{\mathrm{pH}_{1}}\left(\mathrm{pH}_{2}-\mathrm{pH}_{2}{ }^{\prime}\right)+\ldots
\end{aligned}
$$

\footnotetext{
$\left.{ }^{2}\right) 47=$ Dampfdruck des Wassers bei $37^{\circ} \mathrm{C}$ in $\mathrm{mm} \mathrm{Hg}$

${ }^{3}$ ) Für $4 \%$ bzw. $8 \%$ Volumenanteil $\mathrm{CO}_{2}$ beï $760 \mathrm{~mm} \mathrm{Hg}$
}

Hierbei sind $\mathrm{pH}_{1}$ ' und $\mathrm{pH}_{2}$ 'zunächst beliebig festgelegte pH-Werte. Trägt man bei konstantem $\mathrm{pH}_{2}$ den Basenüberschuß als Funktion von $\mathrm{pH}_{1}$ auf und zeichnet die Tangente an die so erhaltene Kurve im Punkt $\mathrm{pH}_{1}{ }^{\prime}$, so erhält man den Differentialquotienten

$\left(\frac{\partial \mathrm{F}}{\partial \mathrm{pH}_{1}}\right)_{\mathrm{pH}_{2}}$ als Steigung dieser Tangente. Ganz analog erhält man den Differentialquotienten $\left(\frac{\partial \mathrm{F}}{\partial \mathrm{pH}_{2}}\right)_{\mathrm{pH}_{1}}$ als

Steigung der Tangente im Punkt $\mathrm{pH}_{2}$ ', wenn man bei konstantem $\mathrm{pH}_{1}$ den Basenüberschuß als Funktion von $\mathrm{pH}_{2}$ aufträgt. $\mathrm{F}\left(\mathrm{pH}_{1}{ }^{\prime}, \mathrm{pH}_{2}\right)$ ergibt sich aus dem Nomogramm als Schnittpunkt der Geraden durch die Punkte $\mathrm{P}_{1}\left(\mathrm{pCO}_{2}, 1, \mathrm{pH}_{1}{ }^{\prime}\right)$ und $\mathrm{P}_{2}\left(\mathrm{pCO}_{2}, 2, \mathrm{pH}_{2}{ }^{\prime}\right)$ mit der Basenüberschußkurve.

Da nur die ersten beiden Glieder der Taylor-Reihe (Gleichung $\mathrm{X}$ ) zur Berechnung des Basenüberschusses verwendet worden sind, hat die so erhaltene Gleichung $\mathrm{BE}=\mathrm{F}\left(\mathrm{pH}_{1}, \mathrm{pH}_{2}\right)$ nur einen beschränkten Gültigkeitsbereich; eine gute Übereinstimmung der so errechneten Basenüberschuß-Werte mit denen, die aus dem Nomogramm entnommen sind, ergibt sich zwischen den Werten $\mathrm{pH}_{1}{ }^{\prime}$ und $\mathrm{pH}_{2}$ '.

Mit den 3 Wertepaaren $\mathrm{pH}_{1}{ }^{\prime}=7,65 ; \mathrm{pH}_{2}{ }^{\prime}=7,45$

$$
\mathrm{pH}_{1}{ }^{\prime \prime}=7,50 ; \mathrm{pH}_{2}{ }^{\prime \prime}=7,30
$$$$
\text { und } \mathrm{pH}_{1}{ }^{\prime \prime \prime}=7,40 ; \mathrm{pH}_{2}{ }^{\prime \prime \prime}=7,20
$$

wurden 3 Gleichungen ermittelt, die die Basenüberschußkurve des Nomogramms in ihrem gesamten Verlauf gut annähern. Die 3 Gleichungen lauten (mit jeweiligem Gültigkeitsbereich, wobei $\mathrm{pH}_{\mathrm{M}}=1 / 2\left(\mathrm{pH}_{1}+\mathrm{pH}_{2}\right)$ :

$\mathrm{BE}_{1}=39 \mathrm{pH}_{1}+19,1 \mathrm{pH}_{2}-432,3$ für $\mathrm{pH}_{\mathrm{M}} \leqslant 7,41$

$\mathrm{BE}_{2}=37 \mathrm{pH}_{1}+31,4 \mathrm{pH}_{2}-506,5$ für 7,41 $<\mathrm{pH}_{\mathrm{M}} \leqslant 7,54$

$\mathrm{BE}_{3}=48 \mathrm{pH}_{1}+36,5 \mathrm{pH}_{2}-628$ für $\mathrm{pH}_{\mathrm{M}}>7,54$

Je nach der Größe von $\mathrm{pH}_{\mathrm{M}}$ wird mit der zugehörigen Gleichung der Basenüberschuß berechnet.

\section{Erläuterungen zum Computerprogramm}

Zur Berechnung der Parameter des Säure-Basen-Haushaltes werden die Gleichungen V, IX und XI benutzt. Wir haben sowohl für den Tischcomputer Monroe $1860^{4}$ ) als auch für die PDP $8^{5}$ ) (in FOCAL) ein Computerprogramm ausgearbeitet. Nach Eingabe der Meßwèrte (Barometerdruck, $\mathrm{pH}_{1}, \mathrm{pH}_{2}$ sowie $\mathrm{pH}_{2}$ ) werden die Parameter $\mathrm{pH}_{\mathrm{a}}, \mathrm{pCO}_{2}, \mathrm{a}$, Standardbicarbonat und Basenüberschuß ausgedruckt. In Abbildung 1 ist ein Ausdruck der PDP 8 wiedergegeben.

\footnotetext{
$\left.{ }^{4}\right)$ Litton Business Systems, Inc., Orange, New Jersey

5 ) integriert im Gemsaec-Fast-Analyzer, Electro-Nucleonics Europe, Stuttgart, Hasenstr. 18
} 


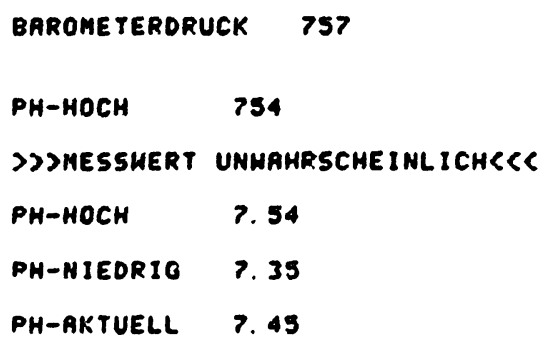

Abb. 1. Ergebnisausdruck eines Säurc-Basen-Status (PDP $8+$ decwriter).

Die vier letztgenannten Parameter werden doppelt geschrieben. Der rechte Teil wird der den Säure-BasenStatus anfordernden Station geschickt, der linke Teil bleibt als Beleg im Laboratorium.

Neben der Berechnung der Parameter des Säure-BasenHaushaltes nach den oben entwickelten Gleichungen enthält das Computerprogramm noch etliche Kontrollen. Ist z. B. die Differenz $\left(\mathrm{pH}_{1}-\mathrm{pH}_{2}\right)$ größer als 0,30 oder kleiner als 0,08 , so wird der Basenüberschuts nicht ausgedruckt. Diese pH-Differenzen entsprechen einer minimalen bzw. maximalen Steigung der $1 \mathrm{~g} \mathrm{pCO} / \mathrm{pH}$ Geraden von - 1 bzw. - 4(3). Treten pH-Differenzen auf, die außerhalb dieser Grenzen liegen, so bedeutet dies, $\mathrm{daß}$ die $\mathrm{pH}$-Messung oder die Blutentnahme fehlerhaft war und eine Wiederholung notwendig ist.

Uber- oder unterschreiten die berechneten Werte die folgenden Grenzen ${ }^{6}$ ), so werden sie mit einer Bemerkung ausgedruckt.

$\mathrm{pH}_{\mathrm{a}}$ : $7,10-7,65$

$\mathrm{pCO}_{2}$, a: $15-80 \mathrm{~mm} \mathrm{HG}$

Standardbicarbonat: $10-40 \mathrm{mmol} / \mathrm{l}$

Basenüberschuß: $(-15)-(+15) \mathrm{mmol} / \mathrm{l}$

6) Diese (irenzen entsprechen 96 Percentilen der ausgewerteten Patientenstichprobe $(n=217)$
Dies soll ein Hinweis dafür scin, daß unter Umständen weitere Kontrollen des Säure-Basen-Status erforderlich sind.

\section{Ergebnisse}

Von 217 Patienten, bei denen die Bestimmung der Parameter des Säure-Basen-Haushaltes durchgeführt wurde, haben wir die Auswertung sowohl graphisch als auch mit Hilfe des oben beschriebenen Computerprogrammes durchgeführt. Bei der graphischen Auswertung der Meßergebnisse wurden $8 \%$ mehr oder minder große Ablesefehler festgestellt, die durch die rechnerische Auswertung vermieden werden können. Die graphische Darstellung der drei errechneten Parameter $\mathrm{pCO}_{2}$, a, Standardbicarbonat und Basenüberschuß als Funktion der gleichen Größen bei manueller Auswertung zeigte eine sehr gute Ubereinstimmung der beiden Verfahren, so daß die hier beschriebene Methode der rechnerischen Auswertung mit Hilfe eines Computers dem bisher üblichen Verfahren zumindest gleichwertig ist, bzw. daß die Nähenungsgleichungen das Siggaard-Andersen-Nomogramm gut wiedergeben. Die Ergebnisse dieser graphischen Darstellungen sind in Tabelle 1 zusammengefaßt.

Die mittleren Abweichungen sind praktisch vernachlässigbar. Die beim Basenüberschuß relativ große maxi- 
Tab. 1. Ergebnisse des Vergleichs von rechnerischer und graphischer Auswertung

\begin{tabular}{lllcl}
\hline Meßgröße & Regressionsgerade & Korrelationskoeffizient & mittlere Abweichung & maximale Abweichung \\
\hline $\mathrm{pCO}_{2}$, a & $\mathrm{y}=-0,19+0,99 \mathrm{x}$ & 0,999 & $-0,68 \mathrm{~mm} \mathrm{Hg}$ & $2 \mathrm{~mm} \mathrm{Hg}$ \\
Standardbicarbonat & $\mathrm{y}=0,99+0,96 \mathrm{x}$ & 0,999 & $-0,16 \mathrm{mmol} / 1$ & $2,2 \mathrm{mmol} / 1$ \\
Basenüberschuß, & $\mathrm{y}=0,06+1,02 \mathrm{x}$ & 0,997 & $0,13 \mathrm{mmol} / 1$ & $2,6 \mathrm{mmol} / 1$ \\
\hline
\end{tabular}

male Abweichung im Vergleich zum $\mathrm{pCO}_{2}$ und Standardbicarbonat hängt mit der Art der Auswertung zusammen, die - wie oben beschrieben - ein Näherungsverfahren ist.

\section{Danksagung}

Herrn J. Hardt danken wir für seine Hilfe.

\section{Literatur}

1. Hardt, J., (1972), Clin. Chem. 18, 658-661.

2. Siggaard-Andersen, O., (1966), The Acid-Base-Status of the Blood, 3. Auflage, Munksgaard, Copenhagen.

3. Astrup, P., (1974), Persönliche Mitteilung.

4. J $\phi$ rgensen, K. \& Astrup, P., (1957), Scand. J. Clin. Lab. Invest. 9, 122-132.
5. Siggaard-Andersen, O., Engel, K., J $\phi$ rgensen, K. \& Astrup, P., (1960), Scand. J. Clin. Lab. Invest. 12, 172-176.

6. Siggaard-Andersen, O., (1962), Scand. J. Clin. Lab. Invest. 14, 598-604.

7. Rowberg, A. \& Lee, St., (1973), Amer. J. Clin. Pathol. 59, 180-184.
Dr. E. Knoll Robert-Bosch-Krkhs. Abt. f. Klin. Chem. 7 Stuttgart 50 Auerbachstr. 110 